\title{
Use of oviduct-inserted acoustic transmitters and positional telemetry to estimate timing and location of spawning: a feasibility study in lake trout, Salvelinus namaycush
}

Thomas R Binder ${ }^{1 *}$, Christopher M Holbrook², Scott M Miehls², Henry T Thompson ${ }^{2}$ and Charles C Krueger ${ }^{3}$

\begin{abstract}
Background: Oviduct-inserted transmitters have shown promise for determining precise location of spawning in fishes. Use of traditional manual tracking to locate expelled oviduct transmitters is laborious and accurate estimates of time of transmitter expulsion require frequent surveys. We tested the feasibility of using oviduct-inserted transmitters with positional telemetry to estimate time and location of spawning in lake trout (Salvelinus namaycush). Three assumptions were tested: (1) oviduct transmitters remain within fish until spawning, (2) oviduct transmitters are expelled with the eggs during spawning, and (3) time and location of oviduct transmitter expulsion can be accurately determined.
\end{abstract}

Results: In the laboratory, 39 of 44 (89\%) lake trout retained an oviduct transmitter until end of the spawning period and all premature transmitter expulsions occurred before maturation. Natural spawning in the laboratory was not feasible; however, of 35 ripe trout that retained transmitters, 31 (89\%) expelled their transmitter with eggs when subjected to manual stripping. Ability to position transmitters with a telemetry array at known spawning sites in Lake Huron (North America) was poor when oviduct transmitters were placed in the substrate compared to transmitters suspended $1 \mathrm{~m}$ above substrate - 78\% of transmitters in substrate could not be positioned. However, in simulations, time and location of spawning were determined with reasonable accuracy by double-tagging trout with one transmitter that is expelled with the eggs during spawning while another transmitter remains in the fish. Accuracy of estimated time and location of transmitter spatial separation varied with distance traveled from spawning site and swimming speed, and was dependent on transmission delay.

Conclusions: Our results satisfied the three assumptions of oviduct tagging and suggested that oviduct transmitters can be used with positional telemetry to estimate time and location of spawning in lake trout and other species. In situations where oviduct transmitters may be difficult to position once expelled into substrate, pairing oviduct transmitters with a normal-sized fish transmitter that remains in the fish is recommended, with spawning inferred when the two tags separate in space. Optimal transmitter delay will depend on expected degree of spawning site residency and swim speed.

Keywords: Acoustic telemetry, Changepoint analysis, Gymnovarian, Spawning behavior, Transmitter retention, Detection probability

\footnotetext{
* Correspondence: tr.binder@gmail.com

'Department of Fisheries and Wildlife, Michigan State University, Hammond

Bay Biological Station, 11188 Ray Rd, Millersburg, MI 49759, USA

Full list of author information is available at the end of the article
} 


\section{Background}

An understanding of the reproductive ecology of fishes is becoming increasingly important in light of widespread ecosystem alterations, such as introduction of exotic species [1-3], critical habitat degradation [4-6], and climate change [7-9]. Fishery managers need to have an understanding of spawning habitat, and the physiochemical and biological variables that promote successful reproduction. Historically, spawning sites have been identified by surveying (for example, visually, by fishing, or hydroacoustics) for aggregations of ripe adult fish [10], which have then allowed researchers to focus on specific areas and to deduce spawning locations at scales of hundreds of meters to kilometers. More recently, advances in biotelemetry have allowed researchers to track the fine-scale movements of individuals during spawning season [11-13]. The current state-of-the-art in animal tracking is positional telemetry - use of three or more stationary receivers to triangulate the precise location of a transmitter-implanted fish to within a few meters of its true location $[14,15]$. In many cases, use of positional telemetry could allow researchers to infer spawning locations at scales of tens to hundreds of meters. Nonetheless, the above techniques have two distinct disadvantages. First, estimation of the precise time of spawning is not possible. Second, the above techniques rely on the assumption that presence of a fish at a given location during the spawning season indicates that an individual spawned at that location. Therefore, additional techniques outside of those described above (for example, sampling egg deposition) are required to determine time of and location of spawning. A possible solution to these limitations is use of oviduct-inserted transmitters to estimate the precise time and location of egg deposition; the idea being that an oviduct transmitter will be expelled from the fish with eggs during spawning, and then located resting on the spawning substrate. The technique was first demonstrated by Pierce [16], who inserted miniaturized radio transmitters into the oviduct of northern pike (Esox lucius) to identify critical shoreline spawning habitat. Since then, the technique has been used successfully in muskellunge, Esox masquinongy [17], and European perch, Perca fluviatilis [18]. In all three of those studies, mobile tracking was used to determine the location of expelled transmitters - Pierce and colleagues $[16,17]$ searched daily for expelled transmitters and Skovrind et al. [18] searched for expelled transmitters twice per week. Because expelled transmitters were not recovered in these studies, it is not possible to assess the accuracy of transmitter expulsion locations determined by mobile tracking. However, Skovrind et al. [18] estimated that accuracy of estimated transmitter locations in their acoustic telemetry study was between 10 and $125 \mathrm{~m}$ dependent on the acoustic environment.
The main disadvantage of using oviduct transmitters in conjunction with mobile telemetry is the amount of labor required to frequently manual track transmitters $[14,15]$. The effort is compounded by the fact that accuracy of estimated time and location of transmitter expulsion will be proportional to the amount of effort expended in locating transmitters - for example, accuracy of the estimated time of spawning will be greater if manual tracking is done daily than if it is done once per week. Positional telemetry using fixed receivers is an attractive alternative to mobile telemetry when the general spawning area (scale of kilometers) is known because positional telemetry provides nearly continuous tracking data $24 \mathrm{~h}$ per day and requires little manual labor beyond deployment and retrieval of the receiver array $[14,15,19]$. In addition, depending on array design, accuracy of position estimates from positional telemetry should be better than those obtained from mobile telemetry [14].

Regardless of tracking method, use of oviduct-implanted transmitters to estimate time and location of egg deposition requires three assumptions: (1) oviduct transmitters remain within fish until spawning (that is, no premature expulsion), (2) oviduct transmitters are expelled with the eggs during spawning, and (3) time and location of oviduct transmitter expulsion can be accurately determined. The biggest unknown with respect to use of oviduct transmitters in conjunction with positional telemetry is the likelihood that the telemetry system will be able to calculate positions of oviduct transmitters after they have been expelled onto or into the substrate. Nonetheless, all three assumptions should be addressed before oviduct transmitters can be deemed a useful tool for studying spawning behaviors in a given species.

Lake trout (Salvelinus namaycush) are an ecologically and economically important fish species in the Laurentian Great Lakes, where they have been the focus of intensive restoration efforts [20,21], and in parts of western United States, where they are invasive [22-24]. Lake trout typically spawn during autumn, and unlike most salmonines, they tend to spawn in open-lake environments $[25,26]$. The reproductive biology of lake trout presents two unique challenges to use of oviduct transmitters in conjunction with positional telemetry. First, unlike the majority of teleost fishes (including northern pike, muskellunge, and European perch), lake trout are gymnovarian, which means that no direct connection exists between the ovary and urogenital pore. Instead, eggs are first released loose into the body cavity and then expelled through the oviduct $[27,28]$. Second, lake trout often spawn on cobble shoals with clean, deep interstitial spaces [25,26]. Expelled oviduct transmitters would likely settle in the spaces between rocks, where their acoustic signals may be blocked and, therefore, less likely to be positioned. In 
this study, we used a series of field and laboratory experiments and random walk models to test the three assumptions of using oviduct transmitters (that is, transmitter retention until spawning, transmitter expulsion during spawning, and ability to accurately determine time and location of transmitter expulsion) to study the spawning behavior of lake trout. Our goal was to assess the potential for using oviduct transmitters in conjunction with positional telemetry to study reproductive ecology, not only in lake trout, but in other fishes as well.

\section{Results}

Given the methodological focus of this study, we recommend that readers review the Methods section at the end of the paper prior to reading Results.

\section{Retention and expulsion of oviduct-inserted transmitters}

In a laboratory study, 48 transmitters were inserted via the oviduct (hereafter 'inserted') or surgically implanted (hereafter 'implanted') into adult female lake trout. Insertion and implantation procedures had no observable effect on the behavior or condition of lake trout during the 10-week lab study; however, four trout died during the study (two from the inserted group: both V7 transmitters; two from the implanted group: one V6 transmitter and one V7 transmitter). Mortalities occurred at 13, 15,36 , and 55 days post tagging, but cause of death was not apparent. Between the two different sized oviduct transmitters tested (V6 and V7, VEMCO, Nova Scotia, Canada), no transmitter-size effect was observed on retention (time-to-event analysis: $\chi^{2}=1.6, P=0.21$ ) or expulsion (Fisher's exact test: $P>0.999$ ), so data from the two tag types were pooled for subsequent analyses to increase statistical power. Data from the four lake trout that died during the study were not included in analyses.

Most lake trout in the inserted and implanted groups retained their oviduct transmitters until the end of spawning season (Table 1). Five (11.3\%) trout expelled their transmitter prematurely. More trout in the inserted group (4 of 22 fish) than the implanted group (1 of 22 fish) prematurely expelled their transmitter. The difference was not significant (survival analysis: $X^{2}=2.0$, $P=0.16$ ), but because our sample size was relatively small, statistical power was marginal (for example, power to identify a $25 \%$ difference in premature expulsion rate was 0.68). All five premature expulsions occurred during the first 2 weeks after tagging, well before maturity and the start of spawning season and ovulation.

Not all lake trout were ovulatory at the end of the spawning season when eggs were manually stripped to determine if oviduct transmitters would be expelled with the eggs. Three trout from the implanted group and one from the inserted group did not shed eggs, nor did they
Table 1 Summary of results from a laboratory study of retention and expulsion of oviduct transmitters in female lake trout

\begin{tabular}{lll}
\hline Stage & Inserted & Implanted \\
\hline Initial sample size (N) & 22 & 22 \\
$\begin{array}{l}\text { Retained oviduct transmitter until end } \\
\text { of spawning period }\end{array}$ & $18(82 \%)$ & $21(95 \%)$ \\
$\begin{array}{l}\text { Became ovulatory (ripened) } \\
\text { Percent of ripe trout that expelled }\end{array}$ & $17(94 \%)$ & $18(86 \%)$ \\
$\begin{array}{l}\text { transmitter with eggs } \\
\text { Percent of total trout that expelled } \\
\text { transmitter with eggs }\end{array}$ & $68 \%$ & $16(89 \%)$ \\
\hline
\end{tabular}

Oviduct transmitters were either 'inserted' into the body cavity via the oviduct or 'implanted' surgically just anterior to the genital pore. Values are the number of lake trout that reached each stage and percentages in parentheses are based on the number of lake trout that made it to the previous stage.

expel the oviduct transmitter. It is possible that these trout would have become ovulatory after a few more days in the holding tank, but any results obtained would have been confounded by previous handling. Therefore lake trout that were not ovulatory were excluded from analysis of transmitter expulsion.

Transmitter expulsion rates during stripping were high for both the inserted and implanted groups $(88 \%$ and $89 \%$, respectively) and did not differ from one another (Fisher's exact test: $P>0.999$ ). The number of stripping motions required to expel the transmitters ranged from 1 to 17 (mean $( \pm \mathrm{SD})=7.0 \pm 3.8$, median $=6$ ) and was not different between the two treatment groups ( $\mathrm{t}$-test: $\mathrm{t}=1.03, P=0.31$ ). In lake trout that did not expel the transmitter during stripping, postmortem autopsies revealed that the oviduct transmitter had moved anteriorly, away from the oviduct.

\section{Estimating time and location of spawning using oviduct transmitters \\ Inferences from a single oviduct transmitter in each fish}

The ability of acoustic receivers to detect stationary transmitters at known lake trout spawning reefs in the Drummond Island Refuge (northern Lake Huron, North America; Figure 1) was reduced when transmitters were manually placed in the substrate to simulate a transmitter expelled during spawning versus a transmitter that was suspended $1 \mathrm{~m}$ above the substrate, simulating a transmitter still within the fish (Figure 2A; linear mixed model: $\mathrm{t}=-13.91, P<0.001)$. Mean $( \pm \mathrm{SE})$ detection probability (that is, number of transmissions detected by receivers as a proportion of total transmissions multiplied by the number of receivers) of control transmitters suspended $1 \mathrm{~m}$ above substrate was $0.74 \pm 0.27$ and $0.70 \pm$ 0.16 for V6 and V7 transmitters, respectively. For transmitters located in the substrate, probability of detection dropped to $0.25 \pm 0.17$ and $0.23 \pm 0.14$ for V6 and V7 transmitters, respectively. Detection probabilities did 


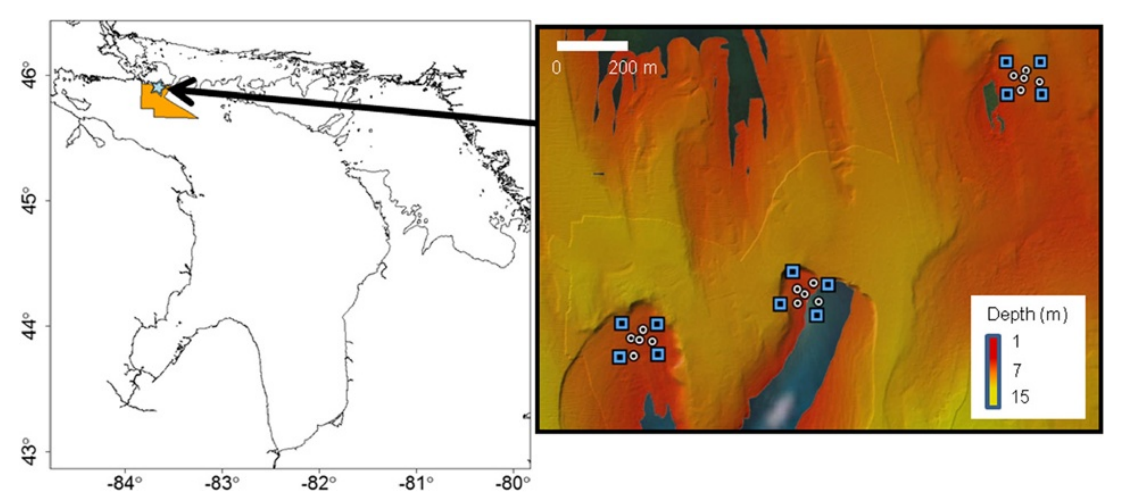

Figure 1 Map of Drummond Island Lake Trout Refuge field study site used for testing the ability of the VEMCO Positioning System (VPS) to position lake trout oviduct transmitters after they are expelled into substrate. Left: Location of the refuge in Lake Huron (North America; shown in orange). The blue star indicates the actual study site (4555.840' N, $83^{\circ} 39.572^{\prime} \mathrm{W}$ ). Right: Location of three small VPS arrays (at known lake trout spawning sites) overlaid on high-resolution multibeam sonar bathymetry. Blue squares indicate location of receivers and white circles represent test transmitter locations. One transmitter at each test transmitter location was suspended $1 \mathrm{~m}$ above the substrate to act as a control and two more were placed in the substrate. A synchronization transmitter was placed the central test transmitter site to synchronize receiver clocks.

not differ between V6 and V7 transmitters (linear mixed model: $\mathrm{t}=-15.56, P=0.38$ ).

The ability of the VEMCO Positioning System (VPS) to calculate positions for transmitters was reduced for transmitters placed in the substrate (Figure 2B; linear mixed model: $\mathrm{t}=-15.56, P<0.001)$ in comparison to suspended transmitters. The decrease in probability of positioning (that is, number of positions obtained as a proportion of total number of transmissions) was greater than the decrease in probability of detection described above (Figure $2 \mathrm{~A}$ and $\mathrm{B})$. Mean $( \pm \mathrm{SE})$ positioning probability for suspended transmitters was $0.69 \pm 0.35$ and $0.64 \pm 0.27$ for V6 and V7 transmitters, respectively. For transmitters placed in the substrate, mean $( \pm \mathrm{SE})$ probability of positioning decreased to $0.04 \pm 0.10$ and
$0.03 \pm 0.10$, respectively. No positions were obtained for $78 \%$ (47 of 60 ) of transmitters in the substrate compared to only $10 \%$ (3 of 30 ) of suspended transmitters that returned no positions. V6 and V7 transmitters did not differ in positioning probability (linear mixed model: $\mathrm{t}=-0.44, P=0.66$ ).

The poor ability of the VPS to position transmitters in the substrate, though many were still being detected by the array (albeit at a lower rate than for suspended transmitters), occurred because transmissions from transmitters planted in the substrate were less likely to be detected simultaneously by three or more receivers. The detection failure by multiple receivers was due, in part, to lower elevation of transmitters in substrate compared to suspended tags and complex rough bathymetry
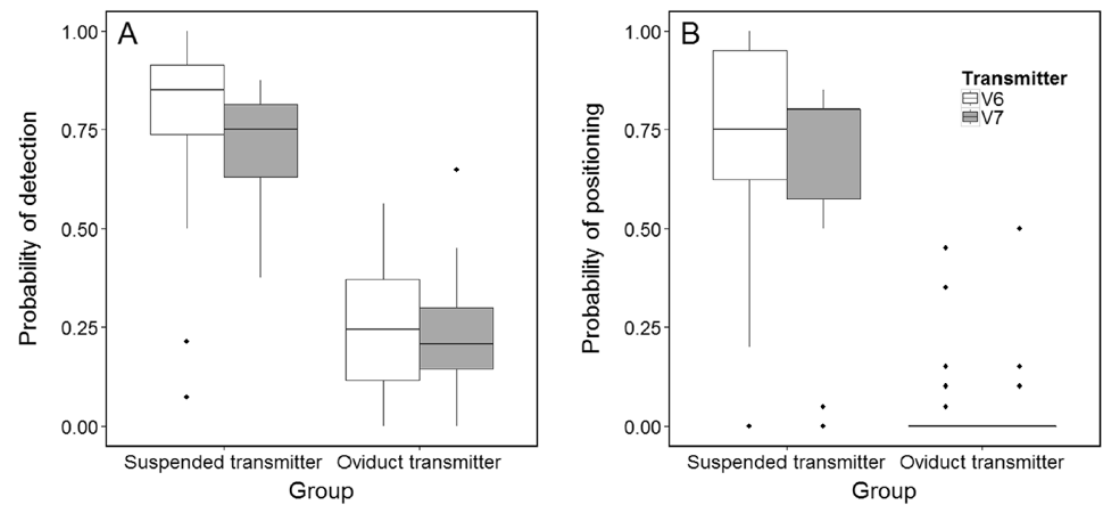

Figure 2 Probability of detecting (A) and positioning (B) V6 and V7 oviduct transmitters (VEMCO, Halifax, Canada) following expulsion into substrate at known spawning sites in the Drummond Island Lake Trout Refuge, Lake Huron. V6 transmitters operated at $180 \mathrm{kHz}$ and V7 transmitters operated at $69 \mathrm{kHz}$. Control transmitters were suspended $1 \mathrm{~m}$ above the substrate. Transmitter type had no significant effect on probability of detection or positioning (linear mixed model: $P<0.001$ ); however, oviduct transmitters in the substrate were significantly less likely to be detected and positioned than control transmitters (linear mixed model; $P<0.001$ for both detection and positioning). Seventy-eight percent of oviduct transmitters in the substrate were not positionable. 
of the cobble substrate in the spawning area, both of which restricted line of sight between transmitters and receivers. Probability of detection was negatively associated with the area obstructing line of sight (cross-sectional area of substrate located above line of sight between transmitter and receiver) between transmitters and receivers (linear mixed model: $\mathrm{t}=2.094, P=0.037$ ). Probability of detection for both suspended transmitters and transmitters in substrate was also negatively associated with distance between transmitter and receiver (linear mixed model: $\mathrm{t}=-8.051, P<0.001$ ).

\section{Pairing oviduct transmitters with larger transmitters to track fish after spawning}

In light of the poor performance of the VPS array with respect to positioning transmitters expelled into the substrate, an alternative paired-transmitter approach was tested via simulation of fish double-tagged with a small oviduct transmitter (to be expelled during spawning) and a larger transmitter that is retained after spawning. Spawning was inferred when the VPS array determined that the two transmitters separated in space (Figure 3). Simulations showed that double-tagging fish can improve estimates of time and location of spawning compared to use of only an oviduct transmitter. Using changepoint analysis [29] on simulated fish tracks, the time and location at which the two transmitters separated (that is, expulsion of oviduct tag during spawning) could be predicted from the change in distance between the two transmitters over time (Figure 4) or by the change in relative positioning probability between the two transmitters over time (Figure 5). Of the two methods, change in relative positioning probability performed best (Figures 4 and 5). On average, location error of estimated transmitter separation was nearly two times greater $(70.1 \mathrm{~m} \mathrm{vs.} 36.7 \mathrm{~m})$ when using change in distance between transmitters than when using change in relative positioning probability. Similarly, average time error of estimated transmitter separation was more than four times greater (10.7 min vs. 2.4 min) when using change in distance between transmitters than when using change in relative positioning probability. The accuracy of estimates of time and location of transmitter separation also varied greatly with characteristics of fish tracks (that is, distance travelled from spawning site (low, medium, medium-high, and high residency groups) and swim speed) and transmitter delay.

\section{Detecting change in distance between paired tags}

When using change in distance between transmitters to estimate time and location of transmitter separation (that is, spawning), location errors ranged from $0.1 \mathrm{~m}$ to $825.2 \mathrm{~m}$. Location error was smaller for fish that remained near the spawning site (high residency group) than for fish that moved farther from the spawning site (low residency group) (Figure 4; general linear model: $\mathrm{t}=-5.512, P<0.001)$. For example, mean $( \pm \mathrm{SE})$ location error was 10 times greater for simulated trout in the low residency group than that for simulated trout in the high residency group $(186.1 \pm 3.7 \mathrm{~m}$ location error $v s .15 .8 \pm$ $0.3 \mathrm{~m}$ location error). Location error also increased with increasing transmission delay, but size of the effect was also greater for fish that moved farther from the spawning site (Figure 4; general linear model: $t=45.940$, $P=<0.001)$. In general, location error increased with swim speed, but size of this effect varied with both distance travelled from spawning site and transmission delay (Figure 4; general linear model: $\mathrm{t}=33.6, P<0.001$ and $\mathrm{t}=10.3, P<0.001$ for interaction with distance travelled from spawning site and transmission delay, respectively). Time errors ranged from $0.0 \mathrm{~min}$ to $569.2 \mathrm{~min}$, but in contrast to location error, decreased with distance travelled from spawning site (Figure 4; general linear model: $\mathrm{t}=-5.51, P<0.001)$. Mean $( \pm \mathrm{SE})$ time error was $6.4 \pm 0.1 \mathrm{~min}$ for simulated trout in the low residency group and 19.2 $\pm 1.0 \mathrm{~min}$ for simulated trout in the high residency group. Like location error, time error was affected by both distance travelled from spawning site and swim speed. Time error increased with increasing transmission delay (Figure 4; general linear model: $t=4.99$, $P<0.001)$. The effect of swim speed on time error, however, was more complicated and was dependent on distance travelled from spawning site (Figure 4; general linear model: $\mathrm{t}=4.00, P<0.001)$.

\section{Detecting change in positioning probability between paired transmitters}

Change in relative positioning probability proved to be more reliable than change in distance between transmitters for estimating the time and location of transmitter separation (that is, spawning). On average, point of transmitter separation was estimated to have occurred within the first two to three fish transmitter transmissions after actual separation. Unlike when using change in distance to estimate transmitter separation, time error did not vary with distance travelled from spawning site (Figure 5; general linear model; $\mathrm{t}=-1.27, P=0.21$ ) or swim speed (Figure 5; general linear model; $\mathrm{t}=-0.52, P=0.60$ ) - mean time error ranged from $2.4 \mathrm{~min}$ to $2.5 \mathrm{~min}$ across all four residency groups (that is, low, medium, medium-high, and high), and from $2.3 \mathrm{~min}$ to $2.5 \mathrm{~min}$ across all levels of swim speed (that is, $0.25,0.50,0.75$, and $1.00 \mathrm{~m} \cdot \mathrm{s}^{-1}$ ). As expected, however, time error did increase significantly with increasing transmission delay (Figure 5; general linear model: $\mathrm{t}=17.01, P<0.001)$. Location error was more variable than time error and was affected by distance travelled from spawning site, transmission delay, and swim speed (Figure 5; general linear model: $\mathrm{t}=-16.11, P<0.001$, $\mathrm{t}=-6.34, P<0.001$, and $\mathrm{t}=-6.34, P<0.001$, respectively). 

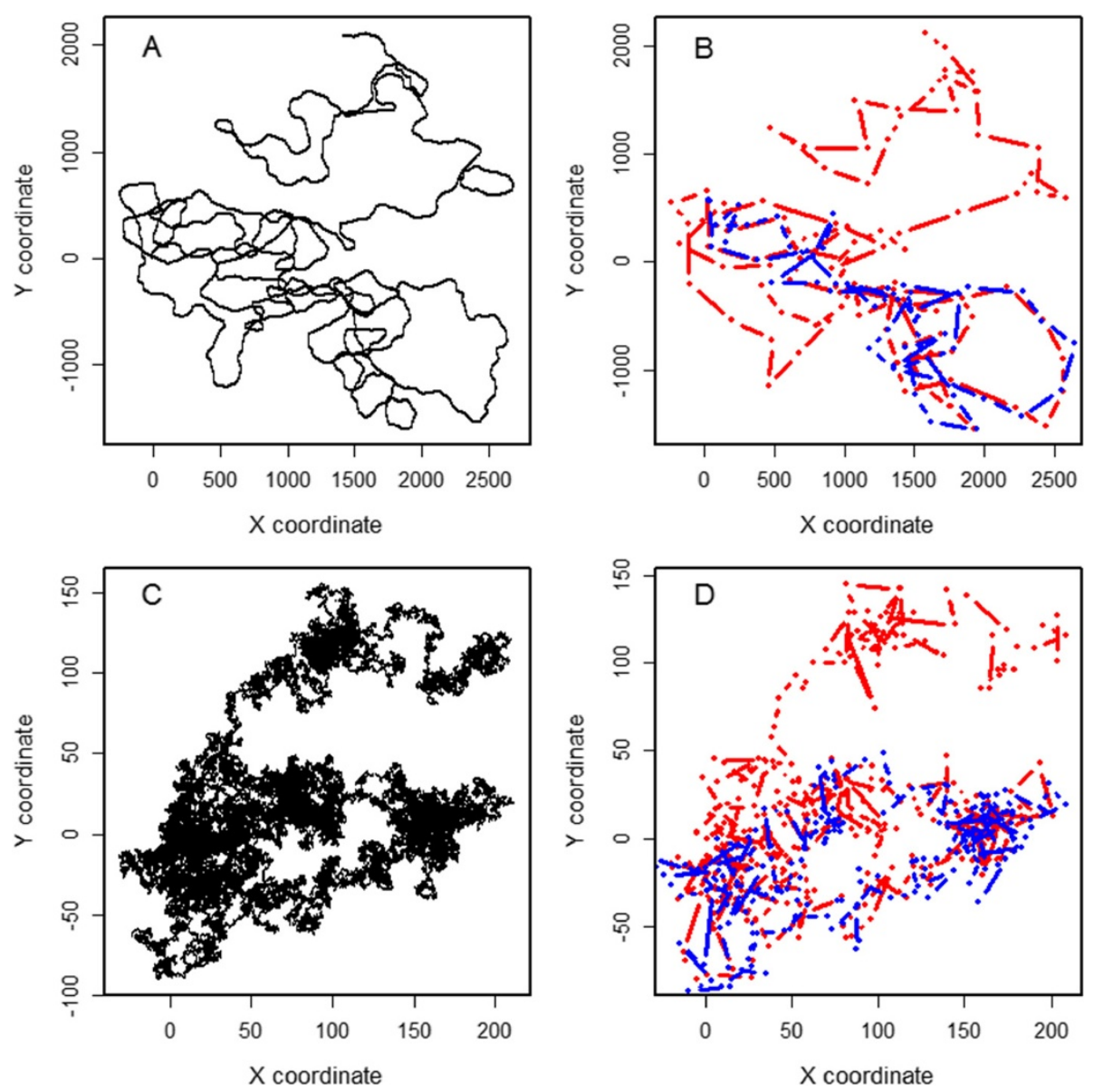

Figure 3 Examples of simulated lake trout tracks (random walk models) and associated transmitter tracks for low-residency (that is, $\sigma=5 ; \mathrm{A}, \mathrm{B})$ and high-residency (that is, $\sigma=90 ; \mathrm{C}, \mathrm{D})$ lake trout. Simulations (6,400 in total) were used to evaluate the potential for use of a paired-transmitter experimental design to estimate the time and location of oviduct transmitter expulsion. Black tracks in (A) and (C) depict the true path of trout, while red and blue tracks in (B) and (D) depict the reconstructed path of the fish transmitter and oviduct transmitter, respectively, based on triangulated positions from a hypothetical positional array. Note the difference in scale between the two trout tracks.

Location errors ranged from $0.2 \mathrm{~m}$ to $995.5 \mathrm{~m}$ and increased with increasing distance travelled from spawning site. For example, mean $( \pm$ SE) location error was $80.7 \pm$ $2.5 \mathrm{~m}$ for simulated trout in the low residency group and $11.1 \pm 0.2 \mathrm{~m}$ for simulated trout in the high residency group. Location error also increased with increasing transmission delay and swim speed, but in both cases the size of the effect was dependent on distance travelled from spawning site (Figure 5; general linear model: $t=25.38$, $P<0.001$ and $\mathrm{t}=20.88, P<0.001$ for interaction with transmission delay and swim speed, respectively). Effect size of swim speed was also dependent on transmission delay (Figure 5; general linear model: $\mathrm{t}=9.80, P<0.001$ ).

\section{Discussion}

Use of oviduct-inserted transmitters to determine time and location of egg deposition represents an important step forward in the use of electronic transmitters to study the reproductive ecology of fishes [30]. Our study is the first to evaluate the potential for use of oviduct transmitters combined with positional telemetry to accurately estimate time and location of spawning in fishes. We tested three assumptions of using oviduct transmitters to determine time and location of egg deposition in lake trout and found that: (1) most oviduct transmitters remained within lake trout until spawning (that is, few were expelled prematurely), (2) most oviduct transmitters were expelled with eggs during manual stripping, and (3) oviduct transmitters were difficult to position once expelled into substrate, but timing and location of transmitter expulsion (indicative of spawning) could be determined with reasonable accuracy in simulations using positional telemetry and a paired-transmitter design where one transmitter is expelled with eggs during spawning and the other remains in the fish.

Transmitter retention and subsequent artificial expulsion rate in lake trout was comparable to those observed in previous studies that have used oviduct transmitters in 

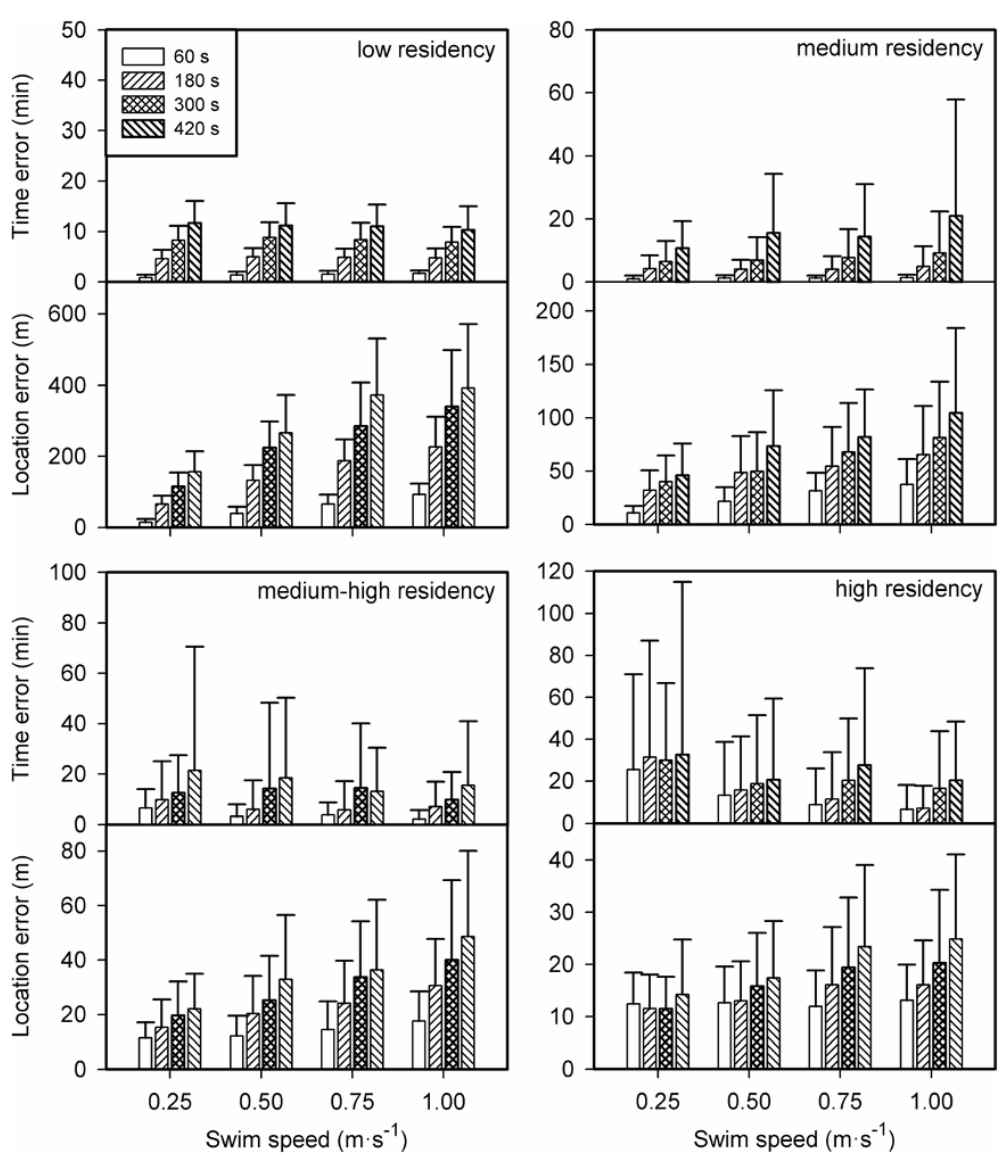

Figure 4 Evaluation of use of change in distance between fish transmitter and oviduct transmitter to estimate time and location of separation in a simulated paired-transmitter study. Panels display data for four different spawning site residency (that is, tendency to remain close to the spawning site) groups, which were simulated using different standard deviation of turn angle in random walk models $(\sigma=5,20,45$, and 90 for low, medium, medium-high, and high residency groups, respectively). Top and bottom graphs in each panel display time error (min) and location error $(\mathrm{m})$, respectively, for point estimates of transmitter separation calculated using changepoint analysis. Shading indicates the effective transmission delay (that is, mean time between transmissions) in seconds.

other fishes. Eighty percent of females that ripened during our study retained the oviduct transmitter until spawning and then expelled it during manual stripping. Pierce et al. [17] reported $60 \%$ and $90 \%$ expulsion in northern pike (Esox lucius) and muskellunge (Esox masquinongy), respectively, and Skovrind et al. [18] noted 92\% expulsion in their study on European perch (Perca fluviatilis). Lake trout have no true connection between the ovary and urogenital pore [27], which might reduce the likelihood that oviduct transmitters would be expelled with the eggs. This condition (gymnovarian) is rare among teleost fishes, but occurs frequently among many of the most commonly studied fish species - that is, trout and salmon (Salmonidae), freshwater eels (Anguillidae), and most non-teleost fishes (for example, sturgeons and lampreys) [28]. All premature expulsions of oviduct transmitters in our study occurred within the first 2 weeks after tagging, well before the onset of the lake trout spawning season. If these premature expulsions had occurred in a field study, likely they would have been recognized as such, and not have confounded results. The cause of premature oviduct transmitter expulsion is not known. Expulsion may have been a passive process, in that transmitters may have been expelled as a result of physical forces at play during normal swimming behavior. Alternatively, the trout may have perceived the transmitter as a foreign object and actively expelled it by muscular contractions or rubbing on the bottom. Some fishes are capable of encapsulating transmitters, and expelling them through the intestine [31,32]. However, given the short time between tagging and premature expulsions, these expulsions likely occurred via the urogenital pore. Either way, the portion of tagged trout that prematurely expelled oviduct transmitters was small enough that, beyond a slight reduction in sample size, impacts on data quality and interpretation would have been minimal.

Most lake trout in our study that retained the transmitter until the end of the spawning period expelled the 

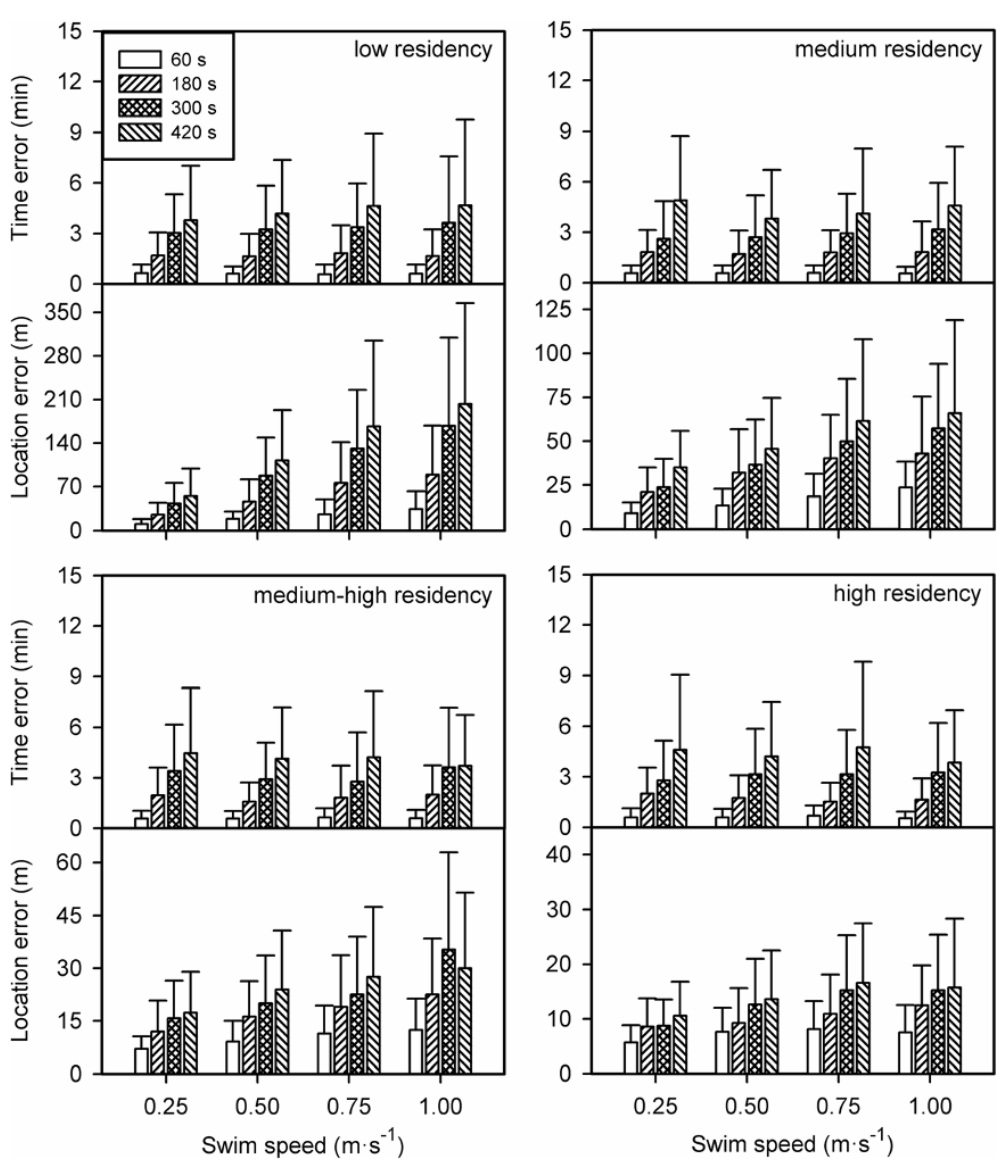

Figure 5 Evaluation of use of change in relative position probability (that is, change in ratio of time since last position) to estimate time and location of separation in a simulated paired-transmitter study. Panels display data for four different spawning site residency (that is, tendency to remain close to the spawning site) groups, which were simulated using different standard deviation of turn angle in random walk models ( $\sigma=5,20,45$, and 90 for low, medium, medium-high, and high residency groups, respectively). Top and bottom graphs in each panel display time error $(\mathrm{min})$ and location error $(\mathrm{m})$, respectively, for point estimates of transmitter separation calculated using changepoint analysis. Shading indicates the effective transmission delay (that is, mean time between transmissions) in seconds.

oviduct transmitter with eggs when manually stripped. However, use of manual stripping as a surrogate for egg deposition during spawning is a source of bias in our study. Although we did our best to approximate normal egg deposition by holding the trout in a natural spawning posture (that is, head and tail flexed slightly upwards) [33,34] during stripping, we do not know to what extent physical forces applied to the eggs and oviduct transmitter during manual stripping represent those present during natural egg deposition. If force applied during manual stripping was greater than would be present during spawning, our results could overestimate transmitter expulsion rate. However, because transmitters were easily expelled, our judgment was that reasonable pressure was applied. Transmitter size (V6 or V7) had no effect on transmitter retention or expulsion rates in our study. Nonetheless, use of the smallest available transmitter that suits the study system and objectives is recommended to minimize the possibility of oviduct blockage [35] and maximize likelihood of transmitter expulsion during spawning.

Poor ability to detect and position transmitters deposited into coarse substrate reduces the usefulness of expelled oviduct transmitters for determining timing and location of spawning. Reef environments with complex topography pose many challenges to acoustic telemetry, including high levels of noise, signal bending, echoes, and signal blocking [36,37]. Line of sight between transmitter and receiver is critical for positioning, so receiver placement in the water column, relative to transmitters, is an important consideration [37,38]. Line of sight may have improved if we had located receivers nearer to the surface, but improvement in line of sight may have come at the expense of increased noise due to wind and waves [36,39] and possibly lower position accuracy due to receiver movement. Line of sight may also have been improved by using more receivers and spacing them closer together. 
In instances where oviduct transmitters are difficult to position once expelled into substrate, a pairedtransmitter design appeared to accurately estimate time and location of spawning. Our decision to use changepoint analysis [29] to determine when the two transmitters separated (that is, began to track differently) was based on a need for objectivity and statistical defensibility. Initially, we attempted to use rule-of-thumb biological filters based on expected behavior of the fish (for example, point at which the distance between fish transmitter and oviduct transmitter was greater than would be expected given the mean swimming speed of the fish). However, results were subjective, and for many trout tracks, rule-of-thumb filters failed even to identify a point of transmitter separation ( $\mathrm{T}$ Binder, unpublished results). In contrast, changepoint analysis produced a point estimate of transmitter separation for all 6,400 trout tracks. One advantage of the changepoint procedure was the change in positioning probability of expelled oviduct transmitters could be used to our advantage and, in our simulation exercise, change in relative positioning probability turned out to be the most accurate method for estimating the time and location of transmitter separation. Ultimately, choice of changepoint method (that is, change in distance between transmitters $v s$. change in relative positioning probability of the transmitters) will depend on how well the acoustic array can position oviduct transmitters after expulsion. For example, in our simulated tracks, positioning probability of oviduct transmitters decreased $90 \%$ after expulsion reflecting the poor ability of the acoustic array to position transmitters in the substrate during our field study. However, in situations where positioning probability of oviduct transmitters decreases only a small amount after expulsion, change in distance between transmitters would likely produce more accurate point estimates of transmitter separation than change in relative positioning probability.

Accuracy of estimated time and location of transmitter separation varied greatly with distance travelled from spawning site (that is, spawning site residency) and swim speed of the fish. In general, time error decreased and location error increased as distance travelled increased from the spawning site. This relationship occurred because fish that travelled further from the spawning site tended to move a greater linear distance from the site of transmitter separation during each transmission interval than fish that tended to remain closer to the spawning site. Time error in estimates of transmitter separation calculated using the change in relative positioning probability method was an exception to the above-stated trend, in that time error was resistant to differences in swimming speed and spawning site residency. The cause for the difference was the fact that positioning rate is a function of transmitter delay and positioning probability, and is independent of the behavior of the fish, except in cases where the fish behaves in a manner that reduces the positioning probability of the transmitter (for example, takes refuge in vegetation [40]).

Transmitters with short delay between transmissions produced more accurate estimates of time and location of transmitter separation, but the effect size of transmitter delay on accuracy of estimates of time and location of transmitter separation was dependent on the behavior of the fish. A tradeoff exists between transmitter delay and the number of transmitters an acoustic array can accommodate at one time. If an acoustic receiver detects a signal from a second transmitter while decoding the signal from the first, the receiver cannot separate the two signals and the result is a code collision, which results in neither transmission being decoded (that is, no detection) [41]. The probability of code collisions increases as transmitter delay decreases and number of transmitters within detection range of the receiver increases [36]. The impact of long transmitter delays on point estimates of transmitter separation was greater in fish with low spawning site residency, indicating that use of a small number of transmitters with a short delay is preferred in these cases. In contrast, because transmitter delay had less impact on estimates of time and location of transmitter separation in fish that showed high spawning site residency, one might choose a long transmitter delay (for example, $5 \mathrm{~min}$ ) to reduce the frequency of transmitter code collisions and allow for more transmitters to be deployed than if a short transmitter delay was chosen. Ultimately, appropriate choice of transmitter delay and number of transmitters deployed will depend on tradeoffs between the desired precision and accuracy of spawning time and location estimates and the behavior of the fish.

\section{Conclusions}

Overall, our results suggest that use of oviduct transmitters with positional telemetry represents a promising new technique for studying spawning behaviors in lake trout. Despite the absence of a direct connection between the ovary and urogenital pore in lake trout, the majority of oviduct transmitters implanted into the body cavity near the oviduct remained within the fish until spawning and were then expelled with eggs during manual stripping. Use of a traditional single-transmitter oviduct tagging study design [16-18] in conjunction with positional telemetry would not be successful in lake trout when spawning occurs over rough stony substrates because oviduct transmitters would be difficult to position. Most transmitters in the substrate continued to be detected on some receivers, but at a low rate. Thus, depending on the size of the study area and the required 
resolution of estimated time and location of spawning, manual tracking would be an alternative option to determine location. However, greater effort would be required to locate transmitters using manual tracking than if the VPS array could be used successfully. We found that use of a paired-transmitter experimental design in conjunction with positional telemetry would be a better solution when oviduct transmitters are expelled into substrates (for example, cobble or mud) that act as barriers to acoustic signal reception. In our simulations, we were able to use the decreased positioning probability of expelled oviduct transmitters to estimate time and location of transmitter separation (that is, spawning) with reasonable accuracy.

Use of oviduct transmitters and positional telemetry to determine time and location of spawning has the potential to be a useful tool for studying reproductive ecology across a broad range of fish species. Beyond the obvious benefits of reduced manual labor and increased precision and accuracy of estimated spawning locations [14], remote monitoring of spawning behavior via positional telemetry may offer opportunities to study spawning in fishes that have previously proven inaccessible [15]. For example, observations of lake trout spawning have been rare because it occurs mainly at night, during a time of year when weather patterns are often unpredictable and dangerous for field work [26]. Nonetheless, effective use of oviduct transmitters to determine time and location of spawning depends on the assumptions that oviduct transmitters will remain within the fish until spawning and then be expelled with eggs at the spawning site, and that oviduct transmitters can be located accurately after expulsion. Failure to meet these assumptions will, at best, reduce sample size (for example, if transmitters are not expelled), and at worst, could result in erroneous conclusions (for example, if transmitters are expelled prematurely). Therefore, regardless of whether researchers use positional telemetry or manual tracking, we recommend that feasibility studies be conducted to test assumptions prior to using oviduct transmitters in a new species.

\section{Methods}

\section{Retention and expulsion of oviduct-inserted transmitters}

We tested the assumptions that oviduct-inserted transmitters are retained until spawning and then expelled with the eggs (Assumptions 1 and 2) in a laboratory tagging study. Forty-eight hatchery-raised, female lake trout (Seneca strain) from Sullivan Creek National Fish Hatchery (Brimley, MI, USA) were transferred to Hammond Bay Biological Station (Millersburg, MI, USA) on 6 August 2013. The trout were housed in a $6,800 \mathrm{~L}$ rectangular $(6.7 \times 1.7 \times 0.9 \mathrm{~m})$ holding tank, supplied with chilled (water temperature between $7^{\circ} \mathrm{C}$ and $\left.13^{\circ} \mathrm{C}\right)$ water from Lake Huron. Trout were offered a diet of $13 \mathrm{~mm}$ brood stock fish pellets until mid-September, when the trout ceased feeding.

Trout were tagged with oviduct transmitters on 17 September 2013. To test the hypothesis that insertion of transmitters through the oviduct does not affect retention and expulsion of transmitters, relative to those that have been surgically implanted, we inserted transmitters via the oviduct in half the fish $(n=24$; hereafter referred to as 'inserted') and surgically implanted transmitters just anterior to the genital pore in the rest $(\mathrm{n}=24$; hereafter referred to as 'implanted'). Half of each tagging group received V6 dummy transmitters (VEMCO, Nova Scotia, Canada; $16.5 \times 6 \mathrm{~mm}, 1 \mathrm{~g}$ in air) and the remaining trout received V7 dummy transmitters (VEMCO; $20 \times 7 \mathrm{~mm}, 1.6 \mathrm{~g}$ in air). Dummy transmitters contained a passive integrated transponder (PIT) tag so that individual fish could be identified. All trout were anesthetized in $40 \mathrm{~L}$ of clove oil solution $(0.8 \mathrm{~mL} / \mathrm{L}$ of $1: 9$ clove oil:ethanol solution) and then placed dorsal side down on a v-board prior to undergoing the tagging procedure. Gills were perfused with aerated lake water throughout the procedures. In trout receiving the transmitter via oviduct-insertion, transmitters were gently inserted through the oviduct and pushed approximately $5 \mathrm{~cm}$ deep using a $10 \mathrm{~cm}$ piece of sterilized $6.5 \mathrm{~mm}$ diameter Tygon tubing. The procedure was performed by a single researcher and took, on average, 20 to $30 \mathrm{~s}$. In trout receiving surgically implanted transmitters, a small incision $(1.5$ to $2.0 \mathrm{~cm}$ ) was made slightly off the ventral midline, approximately $5 \mathrm{~cm}$ anterior to the genital pore. After the transmitter was inserted into the body cavity, the incision was closed using two simple, interrupted sutures (Ethicon, Inc.; 3-0 polydioxanone monofilament). Surgeries were performed by a single surgeon and took, on average, 2 to $3 \mathrm{~min}$. Following tagging, trout were returned to the holding tank to recover from anesthesia.

The holding tank was searched daily, September to November, for expelled oviduct transmitters, as well as expelled loose eggs that would indicate trout were ovulating. During daily searches, the fish were assessed for signs of odd behavior (for example, failure to respond to the researcher's movements or inability to maintain equilibrium) and signs of poor health (for example, dermal discoloration or presence of fungus). If a transmitter was found, the associated PIT tag number, date, and time were recorded. Lake trout did not spawn naturally under laboratory conditions, so manual stripping was used as a surrogate to determine if transmitters would be expelled with eggs. Our assumption was that, while not identical to natural spawning, the manual stripping procedure was representative of the processes that occur naturally during egg expulsion. Manual stripping was conducted on 25 November 2013, to coincide with the end of the Lake Huron lake trout spawning period. The 
ovulating trout were removed from the holding tank one at a time and anesthetized as before to prevent excessive stress. Anesthetized trout were held dorsal side up with heads and tails pointed slightly upward, as occurs during natural egg deposition [33,34]. Their bellies were then massaged from anterior to posterior until the oviduct tag was expelled, or the trout stopped expelling eggs.

Differences in retention of oviduct transmitters were compared using time-to-event analysis ( $\mathrm{R}$ package 'survival'; $\alpha=0.05$ ), which allowed us to not only compare the proportion of individuals that retained the transmitter, but also the temporal pattern of premature expulsion. The proportion of lake trout that expelled the oviduct transmitter during manual stripping was compared among groups using Fisher's Exact Test ( $\mathrm{R}$ package 'stats'; $\alpha=0.05$ ), and the number of stripping motions required to expel the transmitters was compared using a t-test ( $R$ package 'stats'; $\alpha=0.05$ ).

\section{Estimating time and location of spawning using oviduct transmitters \\ Inferences from a single oviduct transmitter in each fish} The ability of the VEMCO Positioning System (VPS) to accurately position expelled oviduct acoustic transmitters in substrate (Assumption 3) was investigated in a field study at known lake trout spawning reefs in the Drummond Island Lake Trout Refuge, northern Lake Huron, North America (Figure 1). Three small VPS arrays (approximately $100 \times 100 \mathrm{~m}$ ) were constructed on three separate near-shore ( 0.6 to $1.3 \mathrm{~km}$ from shoreline) spawning sites. The three sites ranged in depth from approximately 1.5 to $7 \mathrm{~m}$ and consisted of rocky substrate. The approximate $100 \mathrm{~m}$ spacing of receivers was based on recommendations from the manufacturer (VEMCO) on the maximum detection range of the less powerful $180 \mathrm{kHz}$ transmitters. Each array consisted of four receiver locations and a centrally-located synchronization transmitter site, which held transmitters used to synchronize the clocks on receivers. Because the V6 and V7 acoustic transmitters transmit at different frequencies, we installed two acoustic receivers (VR2W-69 kHz and VR2W-180 $\mathrm{kHz}$ ) at each receiver location on a single mooring. The upper receiver was positioned upside down so that the top-mounted hydrophones on the two receivers were located as closely as possible to one another.

Five test transmitter locations were chosen for each VPS array. The first was at the center of the array. The remaining four were scattered throughout the array so that they were located at random distances from the center of the array. Three transmitters of each type (V6 and V7) were deployed at each site. Transmitters were programmed to transmit with a fixed delay of $1 \mathrm{~min}$ and initialization of transmitters was staggered by $20 \mathrm{~s}$ to ensure that no signal code collisions would occur between transmitters. One of each transmitter type was suspended $1 \mathrm{~m}$ above the substrate to act as a control, representing a transmitter that was still within the trout. The two remaining transmitters of each type were placed in the cobble substrate (5 to $20 \mathrm{~cm}$ in diameter) by scuba divers to represent transmitters that had been expelled during spawning. Divers ensured that the transmitters were placed in the space between rocks, as this is most likely how the negatively-buoyant transmitters would settle when expelled naturally during a spawning event. Test transmitters were left in place for $20 \mathrm{~min}$ (allowing for a total of 20 transmissions from each transmitter) before being retrieved and moved to the next test transmitter site. At the end of the study, receivers were retrieved and downloaded, and log files from the receivers were sent to VEMCO for postprocessing using their proprietary hyperbolic positioning algorithms [42].

Probabilities of detecting and positioning control and oviduct transmitters were compared across transmitter type using linear mixed models ( $\mathrm{R}$ package 'Ime4'; $\alpha=$ $0.05)$. Treatment (control transmitter or oviduct transmitter) and transmitter type (V6 or V7) were fixed effects and transmitter identification code and array number were random effects. Variables related to poor detectability of transmitters in the substrate were also investigated using a linear mixed model ( $\mathrm{R}$ package 'lme4'; $\alpha=0.05$ ). Distance between transmitter and receiver, total area of obstruction between transmitter and receiver, tag type (V6 or V7), and treatment (control transmitter or oviduct transmitter) were fixed effects; transmitter identification code and receiver serial number were random effects. Area of obstruction, the total cross-sectional area of substrate located at higher altitude than the line of sight between transmitter and receiver, was calculated using high-resolution $(1 \mathrm{~m}$ horizontal, $10 \mathrm{~cm}$ vertical) multibeam sonar bathymetry (Seabat 7101 system, Teledyne RESON Inc.).

\section{Pairing oviduct transmitters with larger transmitters to track fish after spawning}

The feasibility of using transmitter separation in a pairedtransmitter design to accurately estimate time and location of oviduct transmitter expulsion (Assumption 3) was tested using random walk models to simulate a variety of possible lake trout spawning behaviors [43]. The pairedtransmitter design called for each simulated trout to be implanted with two transmitters; a small oviduct transmitter that would be expelled with the eggs during spawning, and a larger fish transmitter that would remain within the fish after spawning. Time and location at which the two transmitters began to behave differently (point of transmitter separation) was used to estimate time and location of spawning.

Each random walk model (referred to hereafter as 'trout track') consisted of 43,200 steps, each $1 \mathrm{~m}$ in length (total 
track $=43.2 \mathrm{~km}$ ). Steps of the trout track were straight lines, with headings sampled from a random distribution with mean turn angle equal to 0 and standard deviation $\sigma$. For simplicity, the models assumed that trout remained within the VPS array for the entire time series. However, in practice, the only requirements are that the oviduct transmitter be expelled in the array and then the trout remain within the array long enough to determine that the two transmitters had separated. Numerous possible spawning behaviors were simulated to determine how different behaviors, spanning a range of species, could affect ability to accurately estimate time and location of spawning using a paired-transmitter design. To determine how degree of spawning site residency affected our ability to accurately estimate the time and location of transmitter separation (that is, spawning location) four different values were used for $\sigma(5,20,45$, and 90; hereafter referred to as low, medium, medium-high, and high residency groups, respectively). Distance travelled from spawning site was inversely related to $\sigma$ (Figure 3A and C). For example, simulated trout with $\sigma=5$ (low residency group) travelled, on average, 17 times further from the spawning site than simulated lake trout with $\sigma=90$ (high residency group). Mean $( \pm \mathrm{SE})$ distance between all positions for an individual and its actual spawning site was $1,976 \pm 536 \mathrm{~m}, 493 \pm 141 \mathrm{~m}$, $223 \pm 63 \mathrm{~m}$, and $116 \pm 33 \mathrm{~m}$ for simulated tracks with $\sigma=$ $5,20,45$, and 90, respectively. We also varied mean swim speed of individual lake trout between $0.25 \mathrm{~m} \cdot \mathrm{s}^{-1}$ and $1.00 \mathrm{~m} \cdot \mathrm{s}^{-1}\left(0.25,0.50,0.75\right.$, or $\left.1.00 \mathrm{~m} \cdot \mathrm{s}^{-1}\right)$ to cover a range of realistic swimming speeds.

Transmitter positions, such as those that would be triangulated using the VPS system, were overlaid on each trout track. The result was two transmitter tracks, one for the fish transmitter and another for oviduct transmitter (Figure 3B and D). Transmitters were programmed to transmit with mean transmission delay of 60,180 , 300 , or $420 \mathrm{~s}$. The exact delay of each transmission was sampled from a uniform distribution with bounds delay - delay $/ 2$ and delay + delay $/ 2$. These delays were assumed to represent effective transmitter delays, which incorporated not only the nominal delay of the transmitters as programmed by the manufacturer, but also any variation due to environmental variables like wave noise and signal code collisions with other nearby transmitters. For the sake of realism, we incorporated positioning error into each of the transmitter positions by sampling from a bivariate normal distribution with mean equal to the true location ( $\mathrm{x}$ and $\mathrm{y}$ coordinates) of the trout at time $t$ (based on trout track) and a standard deviation of $15 \mathrm{~m}$. At the halfway point of each trout track, the oviduct transmitter was expelled into the substrate as a simulated spawning event. Based on our field test of the ability of the VPS system to position transmitters expelled into the substrate, we assumed the probability of positioning the oviduct transmitter in the substrate from this point forward was reduced to $10 \%$ (that is, only every 10th transmission could be positioned).

In total, 100 trout tracks were simulated for each combination of $\sigma$, swim speed and transmission delay $(6,400$ trout tracks in total). We used the $\mathrm{R}$ statistical package 'changepoint' to estimate time and location of transmitter separation for each simulated trout track [29]. Changepoint analysis, an emerging tool in movement ecology $[44,45]$, uses maximum likelihood methods to determine points in a time series where the mean, variance, or mean and variance of a response variable change. In our analyses, we tested for a single changepoint, and visual inspection of results indicated that the 'changepoint' function 'cpt.meanvar' (that is, changepoint of mean and variance; number of changepoints $(\mathrm{Q})=1$, test.stat $=$ 'Normal') was best able to identify when the two transmitters separated.

We tested two separate methods for estimating time and location of transmitter separation. The first method was to test for a change in the distance between the two transmitters. For each time in the fish transmitter track, we used linear interpolation to estimate the location of the oviduct transmitter at that time. The linear distance between the fish transmitter and oviduct transmitter was calculated and changepoint analysis was used on those values to determine when in the time series the mean and variance of the distance between the two transmitters changed. The second method took advantage of the reduction in positioning probability of expelled oviduct transmitters that we observed in our field trial. For each time in the fish transmitter track, we calculated the ratio of the time since the previous position for the oviduct transmitter and the time since the previous position for the fish transmitter (that is, time since last oviduct transmitter position / time since last fish transmitter position). Changepoint analysis was then used to determine when in the time series the mean and variance of that ratio changed. Change in ratio of positioning probability was used rather than simply testing for a change in the positioning probability of the oviduct transmitter itself because positioning probability can vary naturally due to environmental variability. The ratio method can accommodate this variation because any environmental variation should affect both transmitters in a similar manner. Both methods outlined above can return estimates of time and location of transmitter separation if the oviduct transmitter continues to be positioned (even at a lower rate). However, only the latter method (change in relative positioning probability) can return an estimate of time and location of transmitter separation if the oviduct transmitter ceases to be positioned after expulsion.

The output of the changepoint analysis was an estimate of the location in the fish transmitter track time 
series where the two transmitters separated (that is, began to behave differently). Two metrics were calculated to evaluate the ability of the paired-transmitter design to accurately estimate the time and location of oviduct expulsion: (1) difference in time (hereafter 'time error') between estimated transmitter separation and true time of spawning (that is, the midway point of the trout track), and (2) distance between estimated location of transmitter separation and true location of spawning (hereafter 'location error'). A series of general linear models ( $R$ package 'stats'; $\alpha=0.05$ ) were used to determine the effects of $\sigma$, transmission delay and swim speed on time and location error of estimated transmitter separation. Effects were modeled separately using both the change in distance between transmitter method and change in relative positioning probability method. Because the effect of spawning site residency on accuracy of transmitter separation estimates was of interest, the continuous variable mean distance travelled from spawning site (that is, mean distance between all positions in a given trout track and its true spawning location) replaced $\sigma$ in the models. Interactions between distance travelled from spawning site and transmission delay, distance travelled from spawning site and swim speed, and transmission delay and swim speed were also included in the models.

\section{Competing interests}

The authors declare that they have no competing interests.

\section{Authors' contributions}

TB participated in all aspects of study conception, experimental design, data acquisition and analysis, and drafted the manuscript. $\mathrm{CH}$ participated in data analysis and manuscript preparation. SM participated in study conception, data collection, and manuscript preparation. HT participated in data collection, analysis, and manuscript preparation. CK participated in study conception, experimental design, and manuscript preparation. All authors read and approved the final manuscript.

\section{Acknowledgements}

We thank E. Larson, L. Lesmeister, D. Operhall, and B. Lamoreux for their technical assistance in the laboratory and field. This work was funded by the Great Lakes Fishery Commission by way of Great Lakes Restoration Initiative appropriations (GL-00E23010-3). This paper is contribution 5 of the Great Lakes Acoustic Telemetry Observation System (GLATOS) and contribution number 1875 of the USGS Great Lakes Science Center. Any use of trade, product, or firm names is for descriptive purposes only and does not imply endorsement by the U.S. Government.

\section{Author details}

${ }^{1}$ Department of Fisheries and Wildlife, Michigan State University, Hammond Bay Biological Station, 11188 Ray Rd, Millersburg, MI 49759, USA. ${ }^{2}$ United States Geological Survey, Hammond Bay Biological Station, 11188 Ray Rd, Millersburg, Ml 49759, USA. ${ }^{3}$ Center for Systems Integration and Sustainability, Michigan State University, 1405 South Harrison Road, 115 Manly Miles Building, East Lansing, Ml 48823, USA.

Received: 29 May 2014 Accepted: 11 August 2014

Published: 4 September 2014

\section{References}

1. Mills EL, Leach JH, Carlton JT, Secor CL: Exotic species and the integrity of the Great Lakes. Bioscience 1994, 44:666-676.
2. Vitousek PM, D'Antonio CM, Loope LL, Rejmánek M, Westbrooks R: Introduced species: a significant component of human-caused global change. New Zeal J Ecol 1997, 21:1-16.

3. Hermoso V, Clavero M, Blanco-Garrido F, Prenda J: Invasive species and habitat degradation in Iberian streams: an analysis of their role in freshwater fish diversity loss. Ecol App/ 2011, 21:175-188.

4. Eby LA, Crowder LB, McClellen CM, Peterson CH, Powers MJ: Habitat degradation from intermittent hypoxia: impacts on demersal fishes. Mar Ecol Prog Ser 2005, 291:249-261.

5. Casatti L, Langeani F, Ferreira CP: Effects of physical habitat degradation on the stream fish assemblage structure in a pasture region. Environ Manage 2006, 38:974-982.

6. Wilson SK, Fisher R, Pratchett MS, Graham NAJ, Dulvy NK, Turner RA, Cakacaka A, Polunin NVC, Rushton SP: Exploitation and habitat degradation as agents of change within coral reef fish communities. Glob Change Biol 2008, 14:2796-2809.

7. Roessig JM, Woodley CM, Cech JJ Jr, Hansen LJ: Effects of global climate change on marine and estuarine fishes and fisheries. Rev Fish Biol Fisher 2004, 14:251-275.

8. Ficke $A D$, Myrick CA, Hansen L: Potential impacts of global climate change on freshwater fisheries. Rev Fish Biol Fisher 2007, 17:581-613.

9. Farrell AP, Hinch SG, Cooke SJ, Patterson DA, Crossin GT, Lapointe M, Mathes MT: Pacific salmon in hot water: applying aerobic scope models and biotelemetry to predict the success of spawning migrations. Physiol Biochem Zool 2008, 81:697-709.

10. Goodyear CS, Edsall TA, Ormsby Dempsey DM, Moss GD, Polanski PE: Atlas of the spawning and nursery areas of Great Lakes fishes, Volume 5. Washington, DC: Lake Huron. U.S. Fish and Wildlife Service; 1982. FWS/OBS-82/52.

11. Eiler $\mathrm{JH}$, Nelson BD, Bradshaw RF: Riverine spawning by sockeye salmon in the Taku River, Alaska and British Columbia. Trans Amer Fish Soc 1992, 121:701-708.

12. Bégout Anras ML, Cooley PM, Bodaly RA, Anras L, Fudge RJP: Movement and habitat use by whitefish during spawning in a Boreal lake: integrating acoustic telemetry and geographic information systems. Trans Amer Fish Soc 1999, 128:939-952.

13. Flavelle LS, Ridgway MS, Middel TA, McKinley RS: Integration of acoustic telemetry and GIS to identify potential spawning areas for lake trout (Salvelinus namaycush). Hydrobiologia 2002, 483:137-146.

14. Espinoza M, Farrugia TJ, Webber DM, Smith F, Lowe CG: Testing a new acoustic telemetry technique to quantify long-term, fine-scale movements of aquatic organisms. Fish Res 2011, 108:364-371.

15. Biesinger Z, Bolker BM, Marcinek D, Grothues TM, Dobarro JA, Lindberg WJ: Testing autonomous acoustic telemetry positioning system for fine-scale space use in marine animals. J Exp Mar Biol Ecol 2013, 448:46-56.

16. Pierce RB: Oviduct insertion of radio transmitters as a means of locating northern pike spawning habitat. N Am J Fish Manage 2004, 24:244-248.

17. Pierce RB, Younk JA, Tomcko CM: Expulsion of miniature radio transmitters along with eggs of muskellunge and northern pike - a new method for locating critical spawning habitat. Environ Biol Fish 2007, 79:99-109.

18. Skovrind M, Christensen EAF, Carl H, Jacobsen L, Møller PR: Marine spawning sites of perch Perca fluviatilis revealed by oviduct-inserted acoustic transmitters. Aquat Biol 2013, 19:201-206.

19. Andrews KS, Tolimieri N, Williams GD, Samhouri JF, Harvey CJ, Levin PS: Comparison of fine-scale acoustic monitoring systems using home range size in demersal fish. Mar Biol 2011, 158:2377-2387.

20. Hansen MJ: Lake trout in the Great Lakes: Basinwide stock collapse and binational restoration. In Great Lakes fisheries policy and management: a binational perspective. Edited by Taylor WW, Ferreri CP. East Lansing, MI: Michigan State University Press; 1999:417-454.

21. Muir AM, Krueger CC, Hansen MJ: Re-establishing lake trout in the Laurentian Great Lakes: past, present, and future. In Great Lakes fisheries policy and management: a binational perspective. 2 nd edition. Edited by Taylor WW, Lynch AJ, Leonard NJ. East Lansing, MI: Michigan State University Press; 2013:533-588.

22. Ruzycki JR, Beauchamp DA, Yule DL: Effects of introduced lake trout on native cutthroat trout in Yellowstone Lake. Ecol Appl 2003, 13:23-37.

23. Koel TM, Bigelow PE, Doepke PD, Ertel BD, Mahony DL: Nonnative lake trout results in Yellowstone cutthroat decline and impacts to bears and anglers. Fisheries 2005, 30:10-19.

24. Martinez PJ, Bigelow PE, Deleray MA, Fredenberg WA, Hansen BS, Horner NJ, Lehr SK, Schneidervin RW, Tolentino SA, Viola AE: Western lake trout woes. Fisheries 2009, 34:424-442. 
25. Marsden JE, Casselman JM, Edsall TA, Elliott RF, Fitzsimons JD, Horn WH, Manny BA, McAughey SC, Sly PG, Swanson BL: Lake trout spawning habitat in the Great Lakes - a review of current knowledge. J Great Lakes Res 1995, 21(Suppl 1):487-497.

26. Muir AM, Blackie CT, Marsden JE, Krueger CC: Lake charr Salvelinus namaycush spawning behaviour: new field observations and a review of current knowledge. Rev Fish Biol Fisher 2012, 22:575-593.

27. Henderson NE: The urinary and genital systems of trout. J Fish Res Board Can 1967, 24:447-449

28. Ganguly S: Reproductive biology in fishes and its diversified physiological mechanisms. Int J Sci Env Tech 2013, 2:401-403.

29. Killick R, Eckley l: changepoint: an $R$ package for changepoint analysis. R package version1.1.1; 2014 [http///CRAN.R-project.org/package=changepoint].

30. Crossin GT, Cooke SJ, Goldbogen JA, Phillips RA: Tracking fitness in marine vertebrates: a review of current knowledge and opportunities for future research. Mar Ecol Prog Ser 2014, 496:1-17.

31. Chisholm IM, Hubert WA: Expulsion of dummy transmitters by rainbow trout. Trans Amer Fish Soc 1985, 114:766-767.

32. Marty GD, Summerfelt RC: Pathways and mechanisms for expulsion of surgically implanted dummy transmitters from channel catfish. Trans Amer Fish Soc 1986, 115:577-589.

33. Esteve M, McLennan DA, Gunn J: Lake trout (Salvelinus namaycush) spawning behaviour: the evolution of a new female strategy. Environ Biol Fish 2008, 83:69-76.

34. Binder TR, Thompson HT, Muir AM, Riley SC, Marsden JE, Bronte CR, Krueger CC: New insight into the spawning behavior of lake trout, Salvelinus namaycush, from a recovering population in the Laurentian Great Lakes. Environ Biol Fish 2014, doi:10.1007/s10641-014-0247-6.

35. Peake S, McKinley RS, Beddow TA, Marmulla G: New procedure for radio transmitter attachment: oviduct insertion. N Amer J Fish Manage 1997, 17:757-762

36. Simpfendorfer CA, Heupel MR, Collins AB: Variation in the performance of acoustic receivers and its implication for positioning algorithms in a riverine setting. Can J Fish Aquat Sci 2008, 65:482-492.

37. Welsh JQ, Fox RJ, Webber DM, Bellwood DR: Performance of remote acoustic receivers within a coral reef habitat: implications for array design. Coral Reefs 2012, 31:693-702.

38. Heupel MR, Semmens JM, Hobday AJ: Automated acoustic tracking of aquatic animals: scales, design, and deployment of listening station arrays. Mar Freshwater Res 2006, 57:1-13.

39. Clements S, Jepsen D, Karnowski M, Schreck CB: Optimization of an acoustic telemetry array for detecting transmitter-implanted fish. N Amer J Fish Manage 2005, 25:429-436.

40. Zamora JCL, García-Berthou E: Preliminary telemetry data on the movement patterns and habitat use of European catfish (Silurus glanis) in a reservoir of the River Ebro, Spain. Ecol Freshwater Fish 2007, 16:450-456.

41. Voegeli FA, Lacroix GL, Anderson JM: Development of miniature pingers for tracking Atlantic salmon smolts at sea. Hydrobiologia 1998, 371/ 372:35-46.

42. Smith F: Understanding HPE in the VEMCO positioning system (VPS). Halifax, NS: VEMCO; 2013.

43. Codling EA, Plank MJ, Benhamou S: Random walk models in biology. J Roy Soc Interface 2008, 5:813-834

44. Gurarie $\mathrm{E}$, Andrews RD, Laidre KL: A novel method for identifying behavioural changes in animal movement data. Ecol Lett 2009, 12:395-408.

45. Madon $B$, Hingrat $Y$ : Deciphering behavioral changes in animal movement with a "multiple change point algorithm-classification tree" framework. Front Ecol Evol 2014, 2:1-9.

doi:10.1186/2050-3385-2-14

Cite this article as: Binder et al:: Use of oviduct-inserted acoustic transmitters and positional telemetry to estimate timing and location of spawning: a feasibility study in lake trout, Salvelinus namaycush. Animal Biotelemetry 2014 2:14.

\section{Submit your next manuscript to BioMed Central and take full advantage of:}

- Convenient online submission

- Thorough peer review

- No space constraints or color figure charges

- Immediate publication on acceptance

- Inclusion in PubMed, CAS, Scopus and Google Scholar

- Research which is freely available for redistribution

Submit your manuscript at www.biomedcentral.com/submit
Ciomed Central 